\title{
Detection of Congestive Heart Failure using Naive Bayes Classifier
}

\section{Dipen Deka}

\begin{abstract}
Congestive heart failure (CHF) is gradually becoming more prevalent due to the stressed lifestyles in modern life. Accurate detection with lower computational complexity and lower cost of diagnosis is a challenge to the researchers in this domain. In this work, I have proposed an approach using naive Bayes algorithm with a lesser number of significantly discriminating features for differentiating the CHF subjects from the normal subjects. The small size of feature sets enhances the computational efficiency and the choice of strong features improves the accuracy. The features are chosen on the basis of p-value of the 2-sample t-test performed between the two types of subjects. Using the p-value, 6 features are selected to train, validate and test the classifier. Publicly available benchmark PhysioNet datasets for congestive heart failure patients and normal subjects are used to carry out the experimentation. This approach is able to provide $100 \%$ classification accuracy as well as sensitivity and specificity of $100 \%$ in identifying CHF patients employing Gaussian naive Bayes algorithm.
\end{abstract}

Keywords: Congestive Heart Failure, Time Domain Analysis, Discrete Wavelet Transform, Naive Bayes Classifier,

\section{INTRODUCTION}

With the escalation of heart patients all across the world possibly due to stressed life, correct and low cost diagnosis is becoming vital to enable proper treatment. Often people die due to the negligence towards the minor health problems or delayed information about the actual cause of the disease, especially in the underdeveloped countries. Congestive heart failure (CHF) is one type of acute heart syndrome, in which the pumping capacity of the heart gets diminished. This is a degenerative disease observed mostly in the aged people or someone who has suffered earlier from strokes [1-3]. From the literature it can be observed that electrocardiography signal (ECG), echocardiography, heart rate variability (HRV) signals are used to detect the CHF [3, 9-13]. Further, machine learning (ML) advancements have been instrumental in the quality and quick diagnosis of various types of diseases. Machine learning techniques are getting improved continuously to tackle various types of data, like data of very big size, very scanty in size, again some are of nonlinear high dimensional type and so on [4-6]. Machine learning classifiers such as, k-nearest neighbor $(\mathrm{KNN})$, support vector machine (SVM), decision tree (DT), classification and

Revised Manuscript Received on February 05, 2020.

* Correspondence Author

Dipen Deka*, Assistant Professor, Central Institute of Technology, Kokrajhar, India. Email: d.deka@cit.ac.in

(c) The Authors. Published by Blue Eyes Intelligence Engineering and Sciences Publication (BEIESP). This is an open access article under the CC BY-NC-ND license (http://creativecommons.org/licenses/by-nc-nd/4.0/) regression tree (CART), naive Bayes network (NB), random forest $(\mathrm{RF})$, convolutional neural network (CNN), and various fuzzy classifiers are widely used in many works to detect different types of heart diseases [1-3,7-13]. Wijbenga et al. [8] have performed an analysis on CHF patients by using parameters including left ventricular ejection fraction (LVEF), HRV triangular index (HTI), systolic pressure, HR and found significant change in these parameters for CHF patients. Arbolishvili et al. [9] have observed depressed HRV in the CHF subjects. Engin [10] compared the CHF data with the normal subject's data by using wavelet transform (WT) based variance measure and detrended fluctuation analysis (DFA) coefficient. He found considerable difference between the readings of CHF patients and normal subjects. However, no ML techniques were used in the works discussed above. Melillo et al. [11] have obtained the specificity of $100.00 \%$ and a sensitivity of $89.74 \%$ using CART algorithm. For classification, they used long term HRV parameters, named root mean squared differences of successive differences (RMSSD), standard deviance of the averages of every 5 minute segments of normal to normal interval (SDANN), and total power calculation using Lomb-Scargle (LS) periodogram. Kuntamalla and Reddy [12] have used heart rate sequential trend analysis for discriminating the $\mathrm{CHF}$ beats from the normal sinus beats. They achieved classification accuracy of $96.68 \%$ in differentiating CHF data from normal heart beats data. They improved the accuracy up to $100 \%$ using dual scale entropy analysis [13]. Again, Hossen and Al-Ghunaimi [14] have obtained accuracy up to $90 \%$ using power spectral distribution based analysis. Isler and Kuntalp [15] used linear discrimination analysis by taking short term HRV features and obtained sensitivity of $86.2 \%$ and the specificity of $92.6 \%$. Afterwards, in another work [16], they have combined the short-term time domain parameters and wavelet entropy measure and thus improved the sensitivity and specificity up to $81.8 \%$ and $98.1 \%$ respectively using KNN classifier. For choosing the best combination of features, they utilized genetic algorithm. Masetic and Subasi [3] have compared the accuracy using different classifier and found $100 \%$ accuracy in random forest classifier. They extracted the HRV features by applying autoregressive Burg method. Recently, adopting a different approach, Porumb et al. [17] have taken the ECG signal instead of HRV signal for their analysis and applied CNN classifier to identify the class discriminative regions in ECG to identify the CHF patients. They have obtained 100\% accuracy in their classification work. However, ECG is more prone to noise which leads to the requirement of robust noise canceller. On the top of that identifying such discriminative region increases computational complexity. On the other hand deriving HRV signal is very easy.

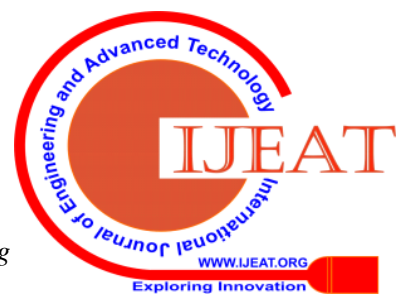


Further, classifiers like ANN, CNN, and RF, SVM techniques are more complex and demanding more computational time. This calls for a simpler but accurate method to identify the CHF cases.

To this end, naive Bayes algorithm is used to test its discrimination power in discerning the CHF patients from normal subjects by extracting features from time domain statistical and discrete wavelet transform (DWT) based analysis. Here, the extracted parameters for both types of data are compared and then their statistical differences were tested. Based on the $p$-value, the features are chosen for NB classifier. Overall, the proposed method provides a simpler, accurate and robust detection of CHF patients.

The remainder of the paper is structured as follows. Section II gives the details of the data used for the work and proposed methodology. In Section III, the findings and its discussion are provided. Finally Section IV concludes the paper.

\section{METHODOLOGY}

\section{A. Data studied}

The RR (R-peak to R-peak of ECG) interval data for the CHF patients and normal subjects are acquired from the benchmark PhysioNet database [18]. The CHF data are retrieved from the Beth Israel Deaconess Medical Center (BIDMC) Congestive Heart Failure Database. The database contains long-term ECG data of 15 subjects (11 men of ages ranging from 22 to 71 years; 4 women, of ages from 54 to 63 years) with major CHF falling under the class 3-4 as per NYHA. The recording of the data were taken before consuming the inotropic agent, milrinone. The ECG signals were sampled at 250 samples/second with 12-bit resolution over a range of \pm 10 millivolts. The original analog recordings were made at the BIDMC using ambulatory ECG recorders with a bandwidth of approximately $0.1 \mathrm{~Hz}$ to $40 \mathrm{~Hz}$.

On the other hand, the control group contains the ECG recordings of normal subjects that are retrieved from the MIT-BIH normal sinus rhythm database. It includes 18 (5 men, of age range 26-45 years, and 13 women, age range 20-50 years) long-term ECG recordings [18]. The subjects were found to have no significant arrhythmias.

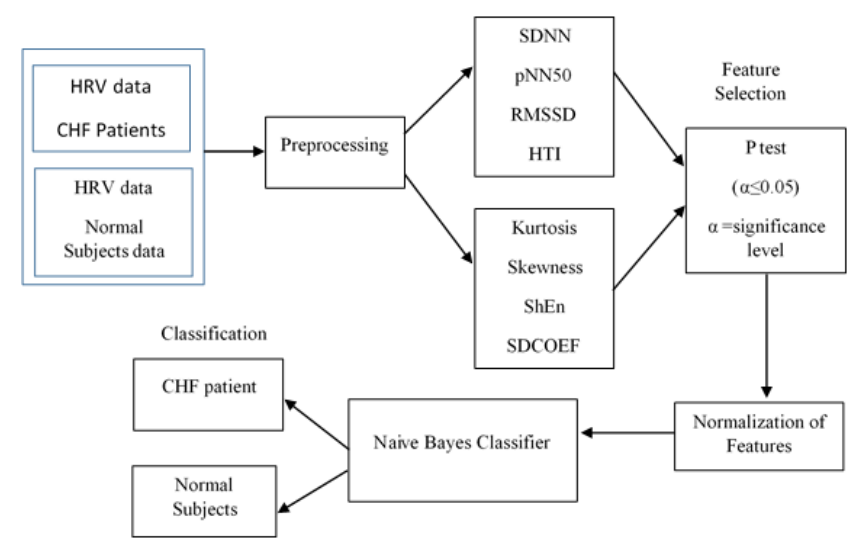

Fig. 1. Block diagram of the proposed methodology

\section{B. Data Preprocessing}

The RR interval data for both the CHF and normal subjects were checked for the presence of outliers. The observed outliers were removed from our analysis as it is found to be less than $1 \%$. The dataset are converted to a format where one variable contains the instances of R-peaks and the other variable shows the corresponding RR intervals. The data will be better termed as HRV time series for our proposed analysis.

\section{Time domain Parameters for evaluating HRV signal}

In this work, the statistical parameters, based on the differences between RR intervals are calculated to observe the pattern of changes in HRV for CHF patients. Time based statistical parameters, namely, standard deviation of normal to normal interval (SDNN), percentage of $\mathrm{NN}$ intervals exceeding $50 \mathrm{~ms}$ (pNN50), root mean square of successive differences (RMSSD), standard deviation of the averages of NN intervals for 5 minute segments (SDANN) and HRV triangular index (HTI) are calculated from the HRV time series for the acquired datasets. It is to specify that $\mathrm{NN}$ intervals means the RR intervals between two normal sinus beats after excluding all the ectopic beats. Then mathematical expressions for calculating these parameters are given in the following equations [19]

$S D N N=\sqrt{\left(\frac{1}{K} \sum_{k=1}^{K}(R R(k)-\overline{R R})^{2}\right)}$

where $\overline{R R}$ indicates the mean value of the RR intervals and $\mathrm{K}$ is the total number of $R R$ intervals.

$N N 50=$ Total number of consecutive $\mathrm{NN}$ intervals whose differences is more than $50 \mathrm{~ms}$.

$$
\begin{aligned}
p N N 50 & =\frac{N N 50}{\text { Total number of NN intervals }} \times 100 \% \\
R M S S D & =\frac{1}{K-1} \sqrt{\sum_{k=1}^{K-1}[R R(k+1)-R R(k)]^{2}} \\
S D A N N & =\sqrt{\left(\frac{1}{L-1} \sum_{l=1}^{L-1}(\overline{S E G(l+1)}-\overline{S E G(l)})^{2}\right)}
\end{aligned}
$$

where $L$ is the number of 5 min segments in whole HRV time series and $\overline{S E G}(l)$ is the average of the NN intervals in a 5 min segment.

and HTI is the ratio of the integration of the density distribution of the NN intervals by the maximum value of density distribution.

\section{Discrete Wavelet Transform (DWT)}

DWT is a discrete version of a time-frequency analysis technique which basically filters a signal by a set of high pass filters (HPF) and low pass filters (LPF) [20,21]. It is suitable for analyzing non-stationary signals since it provides a more localized time-frequency analysis. The increase in the decomposition level provides the information of more detailed (high frequency) components of a signal. The scaling function provides the coarse information, whereas the wavelet function, also known by mother wavelet gives the detailed information of a signal to be analyzed. If $(\psi)_{l, m}(n)$ is a wavelet function, the DWT of a signal $\mathrm{x}[\mathrm{n}]$ is given by

$$
W_{\psi}(l, m)=\frac{1}{\sqrt{M}} \sum_{n=1}^{N} x[n] 2^{l / 2} \psi\left(2^{l} n-m\right)
$$

where $l$ and $m$ are scaling and translation parameter respectively. The scaling parameter can be expanded or contracted to have the frequency or time localized analysis as per requirement. 
For better analysis of a signal, orthonormal wavelet basis function should be smooth and regular. Further the property of compact support is very desirable for leakage free spectral analysis.

For our analysis 'db4' wavelet member of Daubechies family with 4th level of decomposition is chosen considering the smoothness, compact support properties of db4. Here, the vanishing moment and decomposition level is chosen in such a way to reduce the computation cost as well as to maintain the desired accuracy. Since HRV signal spectra lies up to 0.4 $\mathrm{Hz}$, 4th level of decomposition will be sufficient in our case. We have calculated the Shannon entropy (ShEn) [22], kurtosis, skewness [21], and the standard deviation of the $3^{\text {rd }}$ level detail component coefficients as the features to be trained in the naive Bayes classifier.

\section{E. Naive Bayes Classifier}

Naive Bayes classifier is governed by Bayes' theorem, which is based on a key idea that all the features derived from a dataset are independent of the other features [23,24]. This is actually a conditional probability model, which provides the probability of identifying a class $\left(C_{k}\right)$ for a given vector random variable $(\mathrm{X})$ with its vector of feature values $\left(x_{i}\right)$, as given by [24]

$P\left(C=C_{k} \mid X=x_{i}\right)=P\left(C=C_{k}\right) \times \frac{P\left(X=x_{i} \mid C=C_{k}\right)}{P\left(X=x_{i}\right)}$

where $P\left(X=x_{i} \mid C=C_{k}\right)$ is the class-conditional probability and the Bayes discriminant function can be given by Eq. 7 .

$D(x)=\arg \max _{C_{k}} P\left(X=x_{i} \mid C=C_{k}\right) P\left(C=C_{k}\right)$

For the classification purpose, Gaussian naïve Bayes model is employed. In this model, for a particular class $C_{k}$, the feature vector data $\left(x_{i}\right)$ are considered to be following normal distribution. From the trained feature vector, $x_{i}$, the classifier will compute the mean $\left(\bar{x}_{l}\right)$ and variance $\left(\sigma_{k}{ }^{2}\right)$ for the class $C_{k}$ sample. Then for a test data $\left(p_{k}\right)$, the probability distribution of $p_{k}$, given a class $C_{k}$ is given by Eq. 8 .

$P\left(x_{i}=p_{k} \mid C_{k}\right)=\frac{1}{\sqrt{2 \pi \sigma_{k}^{2}}} e^{-\frac{v_{k}-\overline{x_{l}}}{2 \sigma_{k}^{2}}}$

It is to mention here that the simplicity and efficacy of this algorithm for small datasets drive me to use it for discerning CHF patients.

\section{F. Statistical Significance Test}

In this work, statistical significance test plays an important role, as only the significant features/parameters based on $p$-value are fed to the classifier. This approach reduces the computational cost as well as enhance the accuracy of classification. 2-sample $t$-test is applied between the CHF patients and the normal subjects for all the individual features. Those features whose $p$-value is less than $5 \%$ are considered as significantly discriminative. For that purpose, initially the t-statistic has to be calculated and then of $p$-value is obtained from the reading $t$-value and degree of freedom (DF) [25]. I have used IBM SPSS statistic 20 software to obtain the $t$ - and $p$-value. However, their computations are given below:

$$
t=\frac{\overline{X_{A}}-\overline{X_{B}}}{\frac{\sqrt{\sigma_{X_{A}}{ }^{2}+\sigma_{X_{B}}{ }^{2}}}{N}}
$$

where $X_{A}$ and $X_{B}$ are the readings of a parameter for the CHF and normal subjects respectively and $\sigma$ indicates the standard deviation. From the size of the dataset, the DF can be obtained as $\mathrm{DF}=N-1$, where $\mathrm{N}$ is the number of observations. Finally $p$-value can be obtained by using t-statistic chart for the corresponding DF [25].

\section{G. Performance Metrics}

For performance evaluation of the classifier, generally the data is cross validated using either $k$-fold validation, hold out or leave one out technique. The suitability of these techniques are based on the size of the dataset and the demand of speed and accuracy. Leave one out is a very rigorous process, which selects only one sample for test, while putting the other samples for training. This leads to high computational cost. In this work, 10 -fold cross validation technique is used to derive the performance metrics such as accuracy, sensitivity, specificity. In 10-fold cross validation method, whole samples are divided into 10 equal parts, from which one part is kept for testing, while the rest $90 \%$ samples are fed into the classifier for training. Based on the outcome of cross validation, the accuracy, sensitivity, specificity and receiver operating characteristic (ROC) are obtained from the data. The parameter accuracy can be defined as the ratio of summation of true positive and true negative to the total number of events, as given by [26]

$$
\text { Accuracy }=\frac{T P+T N}{T P+T N+F P+F N}
$$

where TP, stands for true positive, which means the presence of an event in both prediction and actual is true.

$\mathrm{TN}$, stands for true negative, which means the absence of an event is true for both prediction and actual.

FP, stands for false positive, which means that an occurrence of an event is predicted but actually the event does not occur. and FN, stands for false negative, which means that the absence of the occurrence of an event is predicted but actually the event does occur.

Accuracy cannot be sufficient to depict the true picture of a model, the information about false positive and false negative events are required to judge the performance of classifier model. Sensitivity of a classifier provides the information about FN events, and it is the given by [26].

$$
\text { Sensitivity }=\frac{T P}{T P+F N}
$$

Again, specificity can be defined as the ratio of the true negative assessment to the actual negative events [26].

$$
\text { Sensitivity }=\frac{T N}{T N+F P}
$$

Besides these metrics, ROC is very important for the assessment of a classifier model because a classifier can be considered as perfectly discriminative, if above a certain threshold, all the abnormal population would come down to zero and below that threshold, all the normal population come down to zero [27].

This ROC characteristics is actually obtained from the plot between specificity (true negative rate) in the $\mathrm{x}$-axis and sensitivity (also known as true positive rate) along y-axis. The absolutely discriminative classifier characteristic will pass though $(0,1)$ coordinate. In other way as the area under this curve (AUC) increases, the performance of a classifier improves. 


\section{Detection of Congestive Heart Failure using Naive Bayes Classifier}

\section{RESULTS AND DISCUSSION}

To perform the classification task, 11 numbers of $\mathrm{CHF}$ subjects from the BIDMC CHF database and 11 numbers of normal subjects from MIT-BIH normal sinus rhythm database are considered. The time domain statistical parameters, SDNN, pNN50, RMSSD, HTI parameters and the DWT based parameters, kurtosis, skewness, Shannon entropy and SD of the wavelet coefficients of $3^{\text {rd }}$ level detail components are obtained from the simulations on MATLAB R2018a environment. Before, time and frequency based evaluations, the outliers in the data are removed. In Table- I, the normalized readings of the HRV parameters for $7 \mathrm{CHF}$ patients and 7 normal subjects are shown. This is done because, fitting the data of $11 \mathrm{CHF}$ and 11 normal subjects in a table takes lots of space. Besides that our motive is to evaluate the $p$-value for the data, so the indicated $p$-value is taken from the whole 22 subjects as used in this work.

Table- I: Normalized readings of HRV features and their $p$-values for CHF patients and Normal subjects

\begin{tabular}{|c|c|c|c|c|c|c|c|c|c|c|c|c|c|c|c|}
\hline 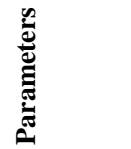 & $\begin{array}{l}\bar{\sigma} \\
\text { 至 } \\
\text { U }\end{array}$ & $\begin{array}{l}\text { 尺ै } \\
\text { 琰 } \\
\text { U }\end{array}$ & $\begin{array}{l}\text { o } \\
\text { 呈 }\end{array}$ & 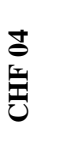 & 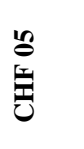 & $\begin{array}{l}\mathscr{8} \\
\text { 至 }\end{array}$ & $\begin{array}{l}\text { 今 } \\
\text { 苞 }\end{array}$ & $\begin{array}{l}\overline{\tilde{J}} \\
\text { है } \\
\text { ż }\end{array}$ & $\begin{array}{l}\frac{N}{\widetilde{J}} \\
\text { हूँ } \\
\text { z }\end{array}$ & $\begin{array}{l}\text { n } \\
\text { ż } \\
\text { ż }\end{array}$ & 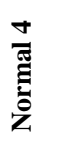 & 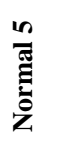 & 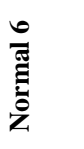 & $\begin{array}{l}\text { N } \\
\text { है } \\
\text { है }\end{array}$ & 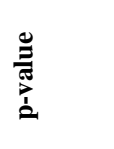 \\
\hline SDNN & 0.38 & 0.25 & 0.23 & 0.11 & 0.08 & 0.40 & 0.31 & 0.61 & 0.81 & 0.44 & 0.24 & 0.80 & 0.62 & 0.33 & $0.0097 *$ \\
\hline pNN50 & 0.01 & 0.14 & 0.16 & 0.08 & 0.03 & 1.00 & 0.08 & 0.09 & 0.14 & 0.14 & 0.13 & 0.35 & 0.26 & 0.19 & 0.674 \\
\hline RMSSD & 0.14 & 0.72 & 0.24 & 0.19 & 0.04 & 0.99 & 0.73 & 0.14 & 0.78 & 0.18 & 0.11 & 0.61 & 0.28 & 0.20 & 0.71 \\
\hline HTI & 0.20 & 0.07 & 0.14 & 0.03 & 0.07 & 0.26 & 0.05 & 0.22 & 0.24 & 0.45 & 0.09 & 0.26 & 0.28 & 0.12 & $0.0375 *$ \\
\hline SDANN & 0.28 & 0.08 & 0.15 & 0.10 & 0.13 & 0.13 & 0.13 & 0.42 & 0.73 & 0.34 & 0.18 & 0.28 & 0.48 & 0.15 & $0.013 *$ \\
\hline ShEn & 0.03 & 0.04 & 0.06 & 0.02 & 0.00 & 0.05 & 0.04 & 0.42 & 0.41 & 0.23 & 0.23 & 0.75 & 1.00 & 0.59 & $0.00015^{*}$ \\
\hline SD_DWT & 0.07 & 0.05 & 0.12 & 0.03 & 0.00 & 0.11 & 0.08 & 0.32 & 1.00 & 0.22 & 0.19 & 0.52 & 0.63 & 0.43 & $0.0014 *$ \\
\hline Kurtosis & 0.09 & 0.03 & 0.04 & 0.24 & 0.28 & 0.02 & 0.10 & 0.02 & 0.05 & 0.01 & 0.02 & 0.01 & 0.00 & 0.00 & $0.0474 *$ \\
\hline Skewness & 0.95 & 0.90 & 0.96 & 0.91 & 0.90 & 0.94 & 0.96 & 0.93 & 0.93 & 0.91 & 0.95 & 0.95 & 0.95 & 0.91 & 0.2337 \\
\hline
\end{tabular}

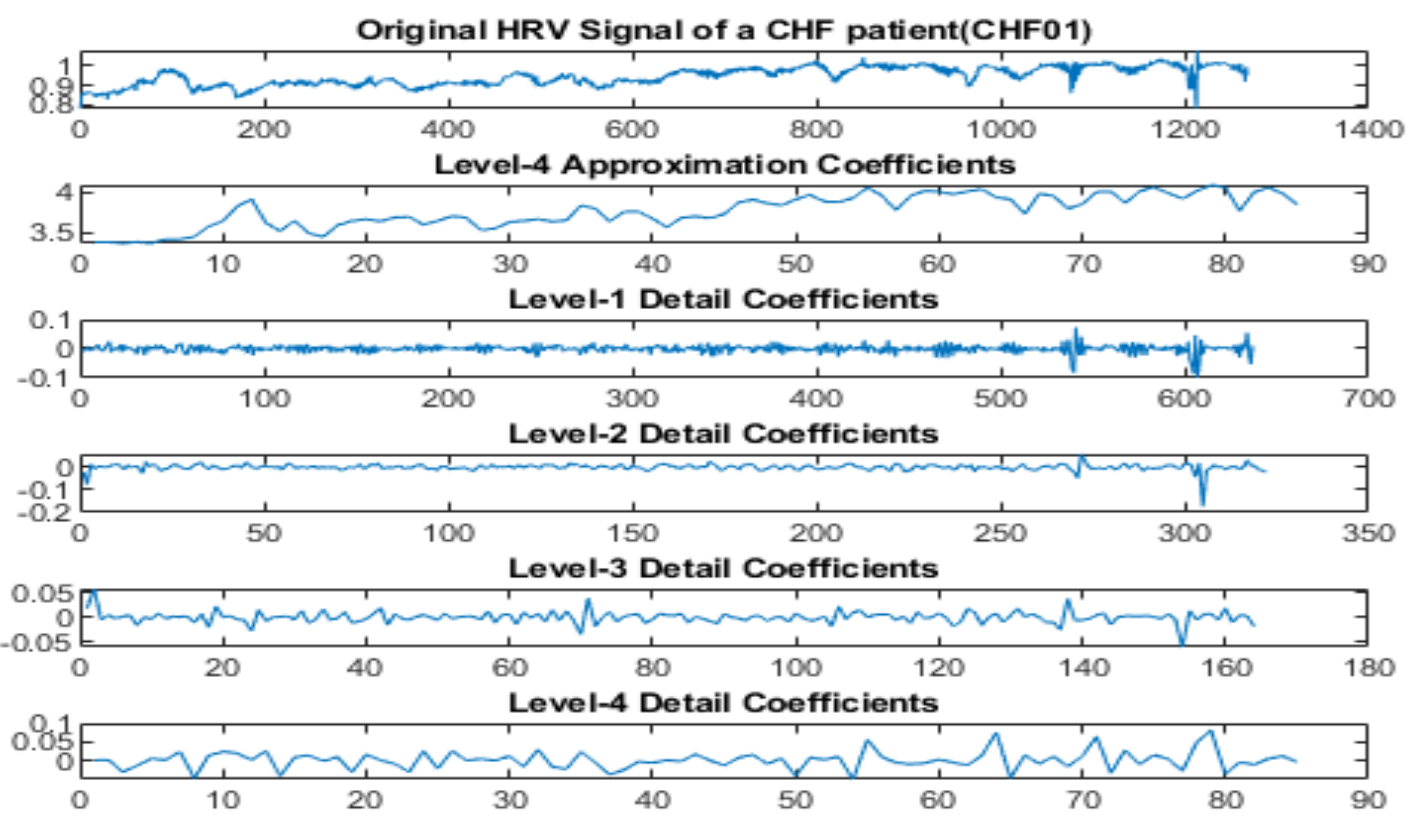

Fig. 2.DWT coefficients for a CHF01 patient 


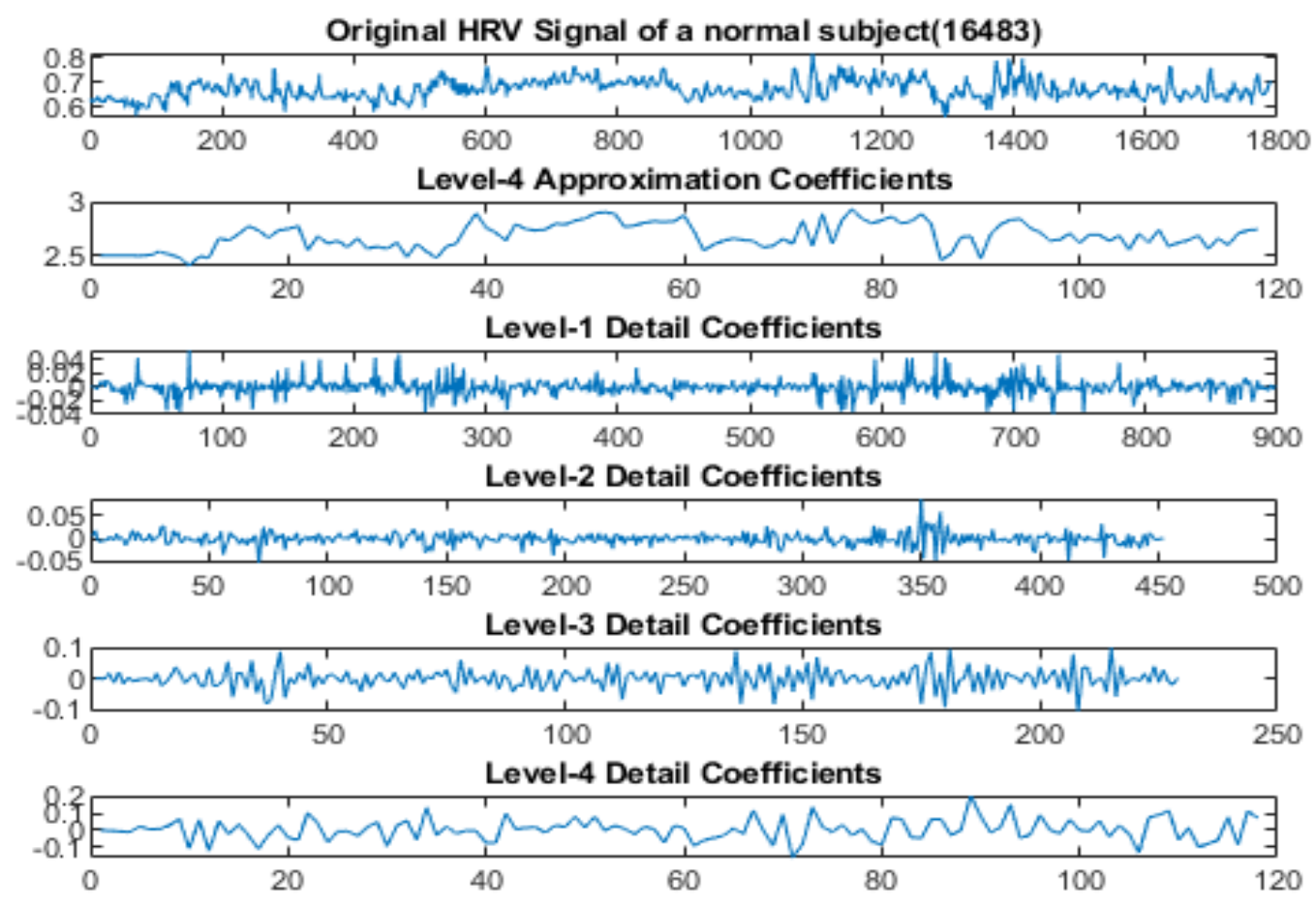

Fig. 3. DWT coefficients for a normal subject

It can be observed from the table that the parameters, pNN50, RMSSD, and skewness does not yield significant differences between the CHF and normal subjects. On the other hand, DWT based parameters ShEn and standard deviation of the DWT coefficients of $3^{\text {rd }}$ level detail component have provided strikingly significant difference. When the data of these two parameters are fed to naive Bayes classifier, the accuracy has improved radically. Further from Fig. 1 and Fig. 2, the difference between the wavelet coefficients are clearly visible.

Table- II: Comparison of accuracy with other works

\begin{tabular}{|c|c|c|}
\hline Authors & Methods & $\begin{array}{l}\text { Accuracy } \\
(\%)\end{array}$ \\
\hline $\begin{array}{l}\text { Isler et al. } \\
(2007)[16]\end{array}$ & k-NN using HRV signal & 96 \\
\hline $\begin{array}{l}\text { Hossen and } \\
\text { Al-Ghunaimi } \\
\text { (2008) [14] }\end{array}$ & $\begin{array}{l}\text { Soft decision tecnique on HRV } \\
\text { signal }\end{array}$ & 88.6 \\
\hline $\begin{array}{l}\text { Kuntamalla } \\
\text { and Reddy } \\
(2010)[12]\end{array}$ & Sequential trend anlysis & 96.68 \\
\hline $\begin{array}{l}\text { Melillo et al. } \\
\text { (2011) [11] }\end{array}$ & CART using HRV signal & 96 \\
\hline $\begin{array}{l}\text { Masetic et al. } \\
\text { (2016) [3] }\end{array}$ & $\begin{array}{l}\text { Random forest algorithm using } \\
\text { HRV signal }\end{array}$ & 100 \\
\hline $\begin{array}{l}\text { Acharya et al. } \\
\text { (2019) [7] }\end{array}$ & $\begin{array}{l}\text { CNN using Very short } 2 \text { sec ECG } \\
\text { signal }\end{array}$ & 98 \\
\hline $\begin{array}{l}\text { Porumb et al. } \\
\text { (2020) [17] }\end{array}$ & CNN using raw ECG & 100 \\
\hline $\begin{array}{l}\text { This proposed } \\
\text { work }\end{array}$ & $\begin{array}{l}\text { Naive Bayes method using HRV } \\
\text { signal }\end{array}$ & 100 \\
\hline
\end{tabular}

The ROC characteristic for the proposed classification model is shown in Fig. 4, which shows the perfect discriminative characteristic for the studied data.

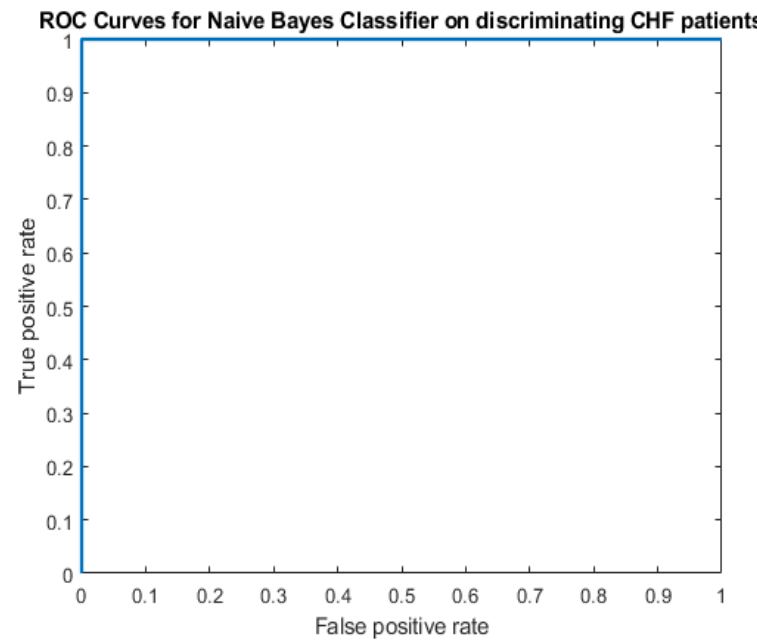

Fig. 4. ROC curve of naïve Bayes classifier in discriminating CHF patients from normal subjects

\section{CONCLUSION}

The proposed method is performing the classification based on only 6 parameters, from the $p$-value assessment which enhances the accuracy as well as the computational efficiency. Besides that, naive Bayes method works very well for small datasets which suits this work, as the number of datasets are quite limited. Another main advantage of naive Bayes, which outweighs other competitive random forest, KNN, SVM algorithms is its simple algorithm. Overall, accuracy, sensitivity, specificity of $100 \%$ is achieved in this work.

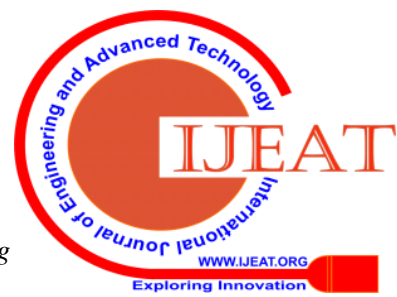




\section{Detection of Congestive Heart Failure using Naive Bayes Classifier}

\section{REFERENCES}

1. D. Levy, M. G. Larson, R. S. Vasan, W. B. Kannel, and K.K. L. Ho, "The Progression From Hypertension to Congestive Heart Failure," The Journal of American Medical Association, vol. 275, no. 20, 1996, pp. 1557-62.

2. K. Swedberg, "Effects of Enalapril on Mortality in Severe Congestive Heart Failure," The New England Journal of Medicine, vol. 316, no. 23, 1987 pp. 1429-35.

3. Z. Masetic, A. Subasi, "Congestive Heart Failure Detection using Random Forest Classifier," Computer Methods and Programs in Biomedicine, vol. 130, 2016, pp. 54-64.

4. E. Alpaydın, Introduction to Machine Learning, 3rd ed., The MIT Press, Cambridge, MA, 2014.

5. C. M. Bishop, Pattern Recognition and Machine Learning, New York: Springer, 2006.

6. S. Sun, "A Survey of Multi-View Machine Learning," Neural Computing and applications, vol. 23, 2013, pp. 2031-2038.

7. U. R. Acharya, H. Fujita, S. L. Oh, Y. Hagiwaral, J. H. Tan, M. Adam, and R. S. Tan, "Deep convolutional neural network for the automated diagnosis of congestive heart failure using ECG signals," Applied Intelligence, vol. 49, 2019, pp.16-27.

8. J. A. M. Wijbenga, A. H. M. M. Balk, S. H. Meij, M. L. Simoons, and M. Malik, "Heart rate variability index in congestive heart failure: relation to clinical variables and prognosis," European Heart Journal, vol. 19, 1998, pp. 1719-1724.

9. G. N. Arbolishvili, V. I. Mareev, I. A Orlova, I. N. Belenkov, "Heart rate variability in chronic heart failure and its role in prognosis of the disease," Kardiologiia, vol. 46, no.12, 2006, pp. 4-11.

10. M. Engin, "Spectral and wavelet based assessment of congestive heart failure patients," Computers in Biology and Medicine, vol. 37, 2007, pp. $820-828$.

11. P. Melillo, R. Fusco, M. Sansone, M. Bracale, and L. Pecchia, "Discrimination power of long-term heart rate variability measures for chronic heart failure detection," Med. Biol. Eng. Comput., vol. 49, 2011, pp. 67-74.

12. S. Kuntamalla and L. R.G. Reddy, "Detecting congestive heart failure using heart rate sequential trend analysis plot," International J. of Engineering Sci. and Technol. vol. 2, no.12, 2010, pp. 7329-7334

13. S. Kuntamalla and L.R.G. Reddy, "Reduced Data Dualscale Entropy Analysis of HRV Signals for Improved Congestive Heart Failure Detection," Measurement Science Review, vol. 14, no. 5, 2014.

14. A. Hossen and B. Al-Ghunaimi, "Identification of Patients with Congestive Heart Failure by Recognition of Sub-Bands Spectral Patterns," World Academy of Science, Engineering and Technology, vol. 44, 2008, pp. 21-24.

15. Y. Isler, M. Kuntalp, "Investigating the effects of wavelet entropy in heart rate variability analysis for diagnosing of congestive heart failure," in: IEEE 14th Signal Processing and Communications Applications Conference (SIU2006), Antalya, Turkey, April 17-19, 2006.

16. Y. Isler and M. Kuntalp, "Combining classical HRV indices with wavelet entropy measures improves to performance in diagnosing congestive heart failure," Computers in Biology and Medicine, vol. 37, pp. $1502-1510,2007$.

17. M. Porumb, E. Iadanzab, S. Massaroc, and L.Pecchia, “A convolutional neural network approach to detect congestive heart failure," Biomedical Signal Processing and Control, vol. 55, 2020.

18. A. L. Goldberger, L. A. N. Amaral, L. Glass, J. M. Hausdorff, P. Ch. Ivanov, R. G. Mark, J. E. Mietus, G. B. Moody, C. -K. Peng, and H. E. Stanley, "PhysioBank, PhysioToolkit, and PhysioNet: Components of a New Research Resource for Complex Physiologic signals," Circulation, vol. 101, no. 23, 2000, pp. e215-e220.

19. M. Malik, "Standards of Measurement, Physiological Interpretation, and Clinical Use," Task Force of The European Society of Cardiology and the North American Society for Pacing and Electrophysiology," Annals of Noninvasive Electrocardiology, vol. 1, no. 2, 1996.

20. S. G. Mallat and S. Zhong, "Characterization of Signals from Multiscale Edges," IEEE Trans. Pattern Anal. Mach. Intell., vol. 14, no. 7, Jul. 1992, pp. 710- 732.

21. A.N. Akansu and M.J.T. Smith, Subband and Wavelet Transforms: Design and Applications, Kluwer Academic Publishers, 1995.

22. C. E. Shannon, "A Mathematical Theory of Communication," The Bell System Technical Journal, vol. 27, 1948, pp. 379-423,623-656.

23. D. D. Lewis, "Naive (Bayes) at forty: The independence assumption in information retrieval," in European Conference on Machine Learning ECML 1998, pp 4-15.

24. I. Rish, "An Empirical Study of the Naïve Bayes Classifier," IJCAI 2001 Workshop Empir. Methods Artif. Intell.
25. C. Redmond and T. Colton, "Clinical significance versus statistical significance," Biostatistics in Clinical Trials.-Wiley Reference Series in Biostatistics (3rd ed.). West Sussex, United Kingdom: John Wiley \& Sons Ltd. pp. 35-36.

26. W. Zhu, N. Zeng, and N. Wang, "Sensitivity, Specificity, Accuracy, Associated Confidence Interval and ROC Analysis," Health Care and Life Sciences, NESIG 2010, pp. 1-9.

27. E. R.DeLong, D. M. DeLong, and D. L. Clarke-Pearson., "Comparing the areas under two or more correlated receiver operating characteristic curves: a nonparametric approach," Biometrics, vol. 44, no. 3, 1988, pp. 837-45.

\section{AUTHORS PROFILE}

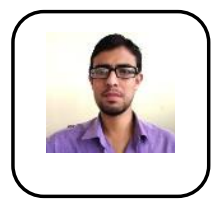

Dipen Deka is an Assistant Professor in the Instrumentation Engineering department of Central Institute of Technology, Kokrajhar, India and presently pursuing Ph.D. in the department of Electronics and Communication Engineering, Tezpur University, India. He received B.E. degree in Instrumentation Engineering from Assam Engineering College and M.Tech. degree in Electronics and Communication Technology from Gauhati University, India in 2005 and 2013, respectively. His research interests include biomedical signal processing, biomedical instrumentation, and transducer \& signal conditioning.

Email: d.deka@cit.ac.in 